\title{
Adverse Events Related to Off-Label Drugs Using Spontaneous Adverse Event Reporting Systems
}

\author{
Nayoung $\operatorname{Han}^{1,2}$ \\ Jung Mi Oh (iD) ${ }^{1,3}$ \\ In-Wha Kim (D)
}

'Research Institute of Pharmaceutical Sciences, Seoul National University, Seoul, Republic of Korea; ${ }^{2}$ College of Pharmacy, Jeju National University, Jejusi, Jeju Special Self-Governing Province, Republic of Korea; ${ }^{3}$ College of Pharmacy, Seoul National University, Seoul, Republic of Korea
Correspondence: In-Wha Kim

Research Institute of Pharmaceutical Sciences, Seoul National University, I

Gwanak-ro, Gwanak-gu, Seoul, 08826,

Republic of Korea

Tel $+822880-7736$

Fax $+822882-9560$

Email iwkim2@hanmail.net
Purpose: The purpose of this study was to investigate the adverse events (AEs) related to the use of off-label drugs.

Materials and Methods: A cross-sectional study was carried out using available data pertaining to off-label drug were sourced from U.S. FDA spontaneous adverse drug reaction reporting database (FAERS) and Korea Adverse Event Reporting System database (KIDSKD) for the years 2014 to 2018. The number and frequencies of AE cases were calculated. Disproportionality was analyzed using the proportional reporting ratio (PRR), the reporting odds ratio (ROR), the information component (IC), and the empirical Bayes geometric mean (EBGM) methods.

Results: The reported AEs associated with off-label drug use were more common among older patients compared with younger patients. Gastric nonspecific symptoms and therapeutic procedure $(4.16-4.57 \%)$ and haemorrage term $(4.16-5.29 \%)$ were the most common $\mathrm{AE}$ symptoms and antithrombotic agents and immunosuppressants were the drugs most commonly reported to cause AEs in FAERS. Secondary term events (43.45-48.62\%) including inappropriate schedule of drug administration and medication error were the most common AEs, and immunosuppressants and antipsychotics were the most common AE-related drugs from KIDS-KD. The numbers of reported AEs in new drug categories such as other antineoplastic agents trended to increase from 2014 to 2018 in both datasets.

Conclusion: The numbers of reported AEs with off-label drug increased annually. AEs associated with off-label drugs may have a significant impact on older patients. Healthcare experts should be concerned about prescriptions of off-label drugs, especially anticoagulants and newly developed drugs such as immunosuppressants and antineoplastic agents.

Keywords: off-label use, adverse events, adverse drug reaction reporting systems, disproportionality

\section{Introduction}

The off-label use of medicine refers to the use of an approved drug for conditions that are not included on the approved label, such as in cases of unapproved indications, age groups, dosages or routes of administration. ${ }^{1}$ Physicians are usually allowed to prescribe approved medications in a manner different from the product label. The off-label use of medicines may be clinically appropriate given the lack of alternative effective approved drugs or the complexity of the patient's condition.

Off-label prescribing is common in several patient populations, especially children, due to the fact that many medications do not receive regulatory approval for the pediatric population. ${ }^{2}$ Therefore, national medicine regulatory authorities have established various strategies and regulations to promote clinical research for 
children and hopefully to decrease off-label drug use. ${ }^{3}$ However, the rate of off-label drug prescribing in children has still increased over the past two decades. ${ }^{4}$ Additionally, antipsychotics have shown the highest rates among off-label drug classes according to previous reports. ${ }^{5-7}$ The use of multiple drugs, often termed polypharmacy in relation to off-label drug use, ${ }^{8}$ especially antipsychotics and antidepressants, has been common in elderly patients. ${ }^{9}$ Therefore, this trend may increase the risk of adverse outcomes in geriatric populations.

On the other hand, large global pharmaceutical companies have traditionally focused on developing medicines for common diseases such as diabetes, cardiovascular disorders, hyperlipidemias, and arthritis, to compensate for their drug discovery efforts and to generate profits. ${ }^{10}$ The market for these classes of medicines given their broad and common indications is currently saturated, which has resulted in competing drug approval processes that increase pricing burdens as well as stricter reimbursement criteria. ${ }^{11}$ Over the past three decades and certainly over the past five years there has a very clear shift to niche markets and personalized medicine. ${ }^{12}$ Compared to these common diseases, rare diseases include many indications where the level of unmet clinical need is very high. There is a lack of approved drug for rare diseases, making it easier to obtain a large market share and pharmaceutical companies get reimbursement for high prices. Therefore, the goal of new drug developments for narrow indications may strengthen at this time. The development of innovative technologies, such as genomics and gene, antisense, and cell therapies, has also accelerated to growth of advanced drugs to treat rare diseases. ${ }^{13}$ Additionally, drugs to treat rare diseases can be considered for marketing approval via conditional rapid authorization systems based on Phase II clinical trial data. Furthermore, certain off-label uses of medicines are integral to disease treatment and are recommended in guidelines. ${ }^{14}$ The off-label use of generic drugs is quite common as a rare disease solution. ${ }^{15,16}$ Therefore, the rate of off-label drug prescribing will likely continue to increase.

Most importantly, the off-label use of medicines can apply to patients in the absence of strong scientific evidence, and patients can be exposed to medicines in offlabel use settings with potential risk. ${ }^{17}$ It has been reported that approximately $73 \%$ of such medicines were estimated to have little or no scientific support, although approximately $21 \%$ of prescriptions overall were reported to be for off-label drug use. ${ }^{7}$ Accordingly, the rate of adverse events (AEs) for off-label use has been reported to be higher than that for on-label use. ${ }^{17}$ Specifically, significant deleterious effects can occur with the off-label use of some drugs, such as in patients with heart valve damage who take fenfluramine ${ }^{18}$ and benfluorex. ${ }^{19}$

Post-market monitoring of drugs is essential for accumulating long-term evidence and for providing assurance regarding the efficacy as well as safety of these drugs. Marketing authorization holders regularly submit periodic safety update reports to medicine authorities, providing new information about the safety of specific drugs. Suspected adverse drug events have been voluntarily reported to spontaneous reporting systems, ${ }^{20,21}$ and these reports include cases of safety issues related to the offlabel use of medicines. ${ }^{9}$ Therefore, the purpose of this study was to investigate the AEs associated with offlabel drug use using the United States Food and Drug Administration (FDA) spontaneous adverse drug reaction reporting database (FAERS) and the Korea Institute of Drug Safety \& Risk Management (Ministry of Food and Drug Safety)-Korea Adverse Event Reporting System database (KIDS-KD).

\section{Materials and Methods Data Source}

Case reports related to off-label use from the FAERS ${ }^{22}$ and KIDS-KD ${ }^{23}$ database were used in this cross-sectional study. The subjects were the patients who were reported to use off-label drugs in both databases. The variables related to patients (gender and age), suspected drug, and AEs were collected. The off-label use and AEs are coded to terms Medical Dictionary for Regulatory Activities (MedDRA, International Council for Harmonisation (ICH) Legal Mention, 2018) ${ }^{24}$ preferred term level terms and as the World Health Organization (WHO) adverse reaction terminology (WHOART version 093) system ${ }^{25}$ in the FARES and KID-KD, respectively. The FAERS data from 2014 to 2018 were downloaded. The data for PRIMARYID that contains off-label use in INDI_PT and a primary suspect drug in ROLE_COD were extracted. The lexicons of the off-label use were based on MedDRA in the FAERS by modification of the previous method $^{26}$ as follows: Off-label use, Product use in unapproved indication, Product use in unapproved therapeutic environment, Product used for unknown indication, Unintentional use for unapproved indication, and Drug effective for unapproved indication. Concomitant drugs 
and AEs in the patients who used off-label drugs were obtained from DRUG and REACT files using the PRIMARYID that contain off-label use, respectively. Offlabel use (Adverse reaction record number (ARRN)/ Sequence number $=1615 / 005$ ) as the WHOART system from 2014 to 2018 in the KID-KD were provided from KIDS. The FAERS and KIRES-KD data are freely accessible, and the requirement for written informed consent from participants was waived because all participants were anonymized by FDA and KIDS and in compliance with the ethical standard. Therefore, no further ethical measure was required.

\section{Data Preprocessing}

Patients in FAERS data were classified into the following age groups according to the format of systems: less than 1 month old, 1 month to 2 years, 2 to 12 years, 12 to 17 years, 17 to 64 years old, and 65 years old or older. ${ }^{27}$ Patients in KIDS-KD data were classified into the following age groups: less than 28 days old, 28 days to 2 years, 2 to 12 years, 12 to 19 years, 19 to 64 years old, and 65 years old or older. ${ }^{28}$ The MedDRA preferred term level terms were combined to Standardised MedDRA Queries (SMQ) due to duplicated reports of variability in the preferred terms chosen to describe the same AE. ${ }^{29}$ Additionally, the WHOART-ARRN terms were grouped into "System Organ Classes (SOC)" level. Duplications were removed according to the FDA recommendation, by selecting the latest FDA_DT (Date FDA received Case) when the CASEIDs were the same and selecting the higher PRIMARYID when the CASEID and FDA DT were the same. ${ }^{30}$ When the drugs were analyzed, only as off-label drugs were used by INDI_DRUG_SEQ for the analysis of drugs in FAERS data. In the KID-KD data, however, only single administered off-label use drugs were extracted, because which drug was administered for off-label use could not be determined when two or more drugs were concomitantly administered. If only trade names were reported, they were converted to generic names. The drugs are classified according to the WHO's Anatomical Therapeutic Chemical (ATC) Classification System.

\section{Data Analysis}

Categorical variables are presented in frequency and percentages. Continuous variables are presented with average and standard variations. The number and frequency of reported AEs or drugs were counted and calculated by the total reported during the period. Disproportionality was analyzed using the proportional reporting ratio (PRR), ${ }^{31}$ the reporting odds ratio
(ROR) ${ }^{32}$ the information component (IC),${ }^{33}$ and the empirical Bayes geometric mean (EBGM) $)^{34}$ methods. The PRR is the degree of disproportionate reporting of an adverse event for a particular drug compared to this same event for all or several other drugs. ${ }^{31}$ A given drug AE pair was defined as a signal if the event count was 3 or more, while the PRR was 2 or more with an associated chi-square value of 4 or more. The ROR is calculated according to the ratio of the odds of the reporting of one specific event versus all other events for a given drug relative to the matching reporting odds for all other drugs. ${ }^{32} \mathrm{It}$ was defined if the lower limit of the $95 \%$ two-sided confidence interval (CI) of ROR exceeded 1. The IC is computed as the logarithm of the ratio of the observed rate of reporting of a specific drug-AE combination to the expected rate. ${ }^{33}$ The AE signal indicated when the lower bound of the $95 \%$ twosided confidence interval of IC (IC025) value exceeds $0 .{ }^{33}$ The EBGM values are adjusted estimates of relative reporting ratios, observed reporting rate/expected reporting rate, after Bayesian multi-item gamma Poisson shrinker. ${ }^{35}$ Safety signals were identified if EBGM and its lower limit of the 95\% confidence interval (EBGM05) values were 2 or more. Data were analyzed with SAS Enterprise Guide version 7.1 (SAS Institute Inc., Cary, NC, USA).

\section{Results \\ Characteristics of the Study Population from FAERS}

The numbers of patients administered with off-label drug and reported AEs in the FAERS data increased annually from 2014 ( $\mathrm{N}=108737)$ to 2018 ( $\mathrm{N}=290458)$. The characteristics of the patients from FAERS are presented in Table 1. The reported $\mathrm{AE}$ frequencies of female patients $(47.02 \%$ to $50.06 \%)$ were higher than those of male patients $(37.13 \%$ to $38.92 \%$ ). The ages of the patients who were reported had AEs ranged from 56.19 to 58.91 years over a course of five years. The AE frequencies of patients less than 17 years of age ranged from $3.10 \%$ (6540 out of 210,789$)$ to $3.68 \%$ (3995 out of 108,737 ), while those who were 65 or older ranged from $26.40 \%(28,702$ out of 108,737$)$ to $28.74 \%$ $(60,571$ out of 210,789$)$. Therefore, reported AEs associated with off-label drug use were more common among older patients compared with younger patients. A range of 3.40 to 3.97 AEs on average was reported for each patient who reported using off-label drugs, with these patients were coadministered with 2.73 to 4.32 other drugs on average. Hospitalization was found as the top-ranked outcomes 
Table I Demographic Characteristics of the Subjects from the FAERS

\begin{tabular}{|c|c|c|c|c|c|}
\hline Characteristics & 2014 & 2015 & 2016 & 2017 & 2018 \\
\hline Number of patients, $\mathrm{n}$ & 108,737 & 180,866 & 210,789 & 237,846 & 290,458 \\
\hline Age, years & $56.19 \pm 21.00$ & $57.86 \pm 20.63$ & $58.91 \pm 20.18$ & $58.23 \pm 20.45$ & $58.33 \pm 20.38$ \\
\hline Less than I month old, $\mathrm{n}$ (\%) & $634(0.58)$ & $1389(0.74)$ & $1239(0.59)$ & $1043(0.44)$ & $1497(0.52)$ \\
\hline I month to 2 years, n (\%) & $684(0.63)$ & $885(0.49)$ & $954(0.45)$ & $\mathrm{I} 00 \mathrm{I}(0.42)$ & $1315(0.45)$ \\
\hline 2 to 12 years, $n(\%)$ & $1577(1.45)$ & $2328(1.28)$ & $2481(1.18)$ & $3174(1.33)$ & $3434(1.18)$ \\
\hline 12 to 17 years, $n(\%)$ & $1100(1.01)$ & $1644(0.91)$ & $1866(0.89)$ & $2405(1.01)$ & $2829(0.97)$ \\
\hline 17 to 64 years old, $n$ (\%) & 40,877 (37.59) & $62,131(34.22)$ & $67,994(32.26)$ & $74,865(31.48)$ & $95,46 \mathrm{I}(32.87)$ \\
\hline 65 years old or older, $\mathrm{n}(\%)$ & $28,702(26.40)$ & $49,74 \mid(27.5 I)$ & $60,57 \mid(28.74)$ & $65,204(27.4 I)$ & $82,042(28.25)$ \\
\hline Unknown, n (\%) & $35,163(32.34)$ & $62,748(34.85)$ & $75,684(35.91)$ & $90,154(37.90)$ & $103,880(35.76)$ \\
\hline \multicolumn{6}{|l|}{ Gender (n, \%) } \\
\hline Male, n (\%) & $42,28 I(38.88)$ & $70,405(38.92)$ & $79,684(37.80)$ & $88,774(37.32)$ & $107,859(37.13)$ \\
\hline Female, $\mathrm{n}(\%)$ & $54,437(50.06)$ & $87,688(48.44)$ & $102,020(48.40)$ & II3,952 (47.91) & $|36,58|(47.02)$ \\
\hline Unknown, n (\%) & $12,019(11.05)$ & $22,773(12.64)$ & $29,085(13.80)$ & $35,120(14.77)$ & $46,013(15.84)$ \\
\hline Co-administration drugs, $\mathrm{n}$ & $2.73 \pm 3.34$ & $3.19 \pm 3.87$ & $3.38 \pm 4.20$ & $4.32 \pm 4.93$ & $3.60 \pm 4.37$ \\
\hline Reported events per patients, $\mathrm{n}$ & $3.4 I \pm 4.24$ & $3.40 \pm 4.43$ & $3.57 \pm 4.65$ & $3.63 \pm 4.64$ & $3.97 \pm 5.19$ \\
\hline Number of outcomes, $n$ & 134,498 & 231,745 & 278,059 & $315,48 \mid$ & 388,969 \\
\hline Congenital Anomaly, n (\%) & $710(0.53)$ & $2186(0.94)$ & $1338(0.48)$ & $1306(0.4 \mid)$ & $1957(0.50)$ \\
\hline Death, n (\%) & $22,191(16.50)$ & $34,532(14.90)$ & $36,349(13.07)$ & $43,366(13.75)$ & $46,401(11.93)$ \\
\hline Disability, n (\%) & $3500(2.60)$ & $4778(2.06)$ & $5283(1.90)$ & $5333(1.69)$ & $6840(1.76)$ \\
\hline Hospitalization - Initial or Prolonged, n (\%) & $41,956(31.19)$ & $74,687(32.23)$ & $91,272(32.82)$ & $99,582(31.57)$ & $|23,30|(3 \mid .70)$ \\
\hline Life-Threatening, n (\%) & $4458(3.31)$ & $6755(2.91)$ & $7878(2.83)$ & $9799(3.11)$ & $14,015(3.60)$ \\
\hline Other Serious (Important Medical Event), n (\%) & 61,581 (45.79) & $108,699(45.90)$ & $135,918(48.88)$ & $156,084(49.47)$ & $196,443(50.50)$ \\
\hline $\begin{array}{l}\text { Required Intervention to Prevent Permanent } \\
\text { Impairment/Damage, } \mathrm{n}(\%)\end{array}$ & $102(0.08)$ & $108(0.05)$ & $21(0.01)$ & II $(0.00)$ & $12(0.00)$ \\
\hline
\end{tabular}

Note: Values are reported as $\mathrm{n}(\%)$ or mean \pm standard deviation.

Abbreviation: FAERS, Food and Drug Administration Adverse Event Reporting System.

(31.19-32.82\%) of AEs followed by death (11.93-16.50\%) except for other serious medical event.

\section{AEs Related to Off-Label Drugs in FAERS}

Gastrointestinal nonspecific symptoms and therapeutic procedures represented the most common SMQ from $2014(4.16 \%)$ to $2018(4.26 \%)$, except for haemorrhage term $(39,870,5.29 \%)$, which was the most common SMQ in 2016, when off-label drugs were administered concomitantly among AE symptoms from the FAERS data (Table 2). The next common SMQ was anticholinergic syndromes $(3.17 \%$ to $4.02 \%)$, followed by neuroleptic malignant syndromes $(3.30 \%$ to $3.53 \%)$.

\section{Off-Label Drugs Related to Reported AEs in FAERS}

AEs related to administered off-label drugs were used for a further analysis. In the FAERS data, the numbers of offlabel drug related to reported AEs increased annually from
$2014(\mathrm{~N}=295556)$ to $2018(\mathrm{~N}=1042836)$ and the ATC classification of the off-label used drugs are presented in Table 3. Antithrombotic agents were the drugs most commonly reported to cause in $2014(18,315$ out of 295,556 , $6.20 \%), 2015$ (36,033 out of 574,499,6.27\%), and 2016 $(50,899$ out of $709,274,7.18 \%)$. Immunosuppressants were the most commonly reported AE drugs in 2017 (81,944 out of $1,026,588,7.98 \%)$, while drugs for peptic ulcer and gastro-oesophageal reflux disease was most commonly reported in relation to AEs in $2018(69,766$ out of $1,042,836,6.69 \%)$. The number of reported AEs in new drug categories, such as immunosuppressants and other antineoplastic agents, tended to increase from 2014 to 2018 compared to other drugs.

\section{Signals Related to Off-Label Drugs in FAERS}

A total of 21,659,660 drug-AE pairs were analyzed by disproportionality for detecting signals. The PRR, ROR, 
Table 2 Top Ten Adverse Event Symptoms of Off-Label Drug Use from the FAERS Data

\begin{tabular}{|c|c|c|c|c|c|}
\hline SMQ & $\begin{array}{c}2014 \\
(N=37 \mid 163)\end{array}$ & $\begin{array}{c}2015 \\
(N=614905)\end{array}$ & $\begin{array}{c}2016 \\
(N=753034)\end{array}$ & $\begin{array}{c}2017 \\
(N=863205)\end{array}$ & $\begin{array}{c}2018 \\
(N=1153963)\end{array}$ \\
\hline $\begin{array}{l}\text { Gastrointestinal nonspecific symptoms and } \\
\text { therapeutic procedures }\end{array}$ & $15,440(4.16)$ & $28,412(4.62)$ & $34,385(4.57)$ & $37,586(4.35)$ & $49,148(4.26)$ \\
\hline Haemorrhage terms (excl laboratory terms) & $|2,83|(3.46)$ & $22,611(3.68)$ & $39,870(5.29)$ & $30,697(3.56)$ & $37,204(3.22)$ \\
\hline Anticholinergic syndrome & $14,935(4.02)$ & $22,425(3.65)$ & $23,880(3.17)$ & $30,844(3.57)$ & $38,704(3.36)$ \\
\hline Neuroleptic malignant syndrome & $12,858(3.46)$ & $21,689(3.53)$ & $25,281(3.36)$ & $30,096(3.49)$ & $38,067(3.30)$ \\
\hline Accidents and injuries & $9630(2.59)$ & $\mid 4,803(2.4 \mid)$ & $20,283(2.69)$ & $22,520(2.61)$ & $28,417(2.46)$ \\
\hline Hyperglycaemia/new onset diabetes mellitus & $856 I(2.31)$ & $|5,54|(2.53)$ & $18,639(2.48)$ & $24,730(2.86)$ & $29,827(2.59)$ \\
\hline Hypersensitivity & $13,184(3.55)$ & $19,630(3.19)$ & $6301(0.84)$ & $7109(0.82)$ & $34,739(3.01)$ \\
\hline Dementia & $6106(1.65)$ & $|0,44|(\mid .70)$ & 12,698 (1.69) & $15,892(1.84)$ & $20,557(1.78)$ \\
\hline Arthritis & 489I (1.32) & 8171 (1.33) & $10,939(1.45)$ & I3,467 (I.45) & $21,062(1.83)$ \\
\hline Peripheral neuropathy & $4736(1.28)$ & 8745 (I.42) & $10,762(1.43)$ & $12,178(1.41)$ & I5,376 (1.33) \\
\hline
\end{tabular}

Note: Values are reported as $n(\%)$.

Abbreviations: FAERS, Food and Drug Administration Adverse Event Reporting System; SMQ, Standardised Medical Dictionary for Regulatory Activities Queries.

IC, and EBGM methods detected the 51,405, 97,854, 143,776, and 23,749 signals, respectively. The 20,869 signals were detected by all these four methods. AEs associated with off-label drugs are listed in Table 4 and Supplementary Table S1. The signal of haemorrhage term was most frequently reported for aspirin, followed by rivaroxaban.

\section{Characteristics of the Study Population from KIDS-KD}

In the KIDS-KD data, the number of patients administered offlabel drugs who also reported AEs in 2014 increased in 2018 (from 408 to 1134). The characteristics of the patients from the KIDS-KD are presented in Table 5. The frequencies of patients less than 19 years of age ranged from $8.09 \%$ (33 out of 408 ) to

Table 3 Off-Label Drug Classifications for Reported Adverse Events in the FAERS Data

\begin{tabular}{|c|c|c|c|c|c|}
\hline Drug Classification & $\begin{array}{c}2014 \\
(N=295556)\end{array}$ & $\begin{array}{c}2015 \\
(N=574499)\end{array}$ & $\begin{array}{c}2016 \\
(N=709274)\end{array}$ & $\begin{array}{c}2017 \\
(N=1026588)\end{array}$ & $\begin{array}{c}2018 \\
(N=1042836)\end{array}$ \\
\hline Antithrombotic agents & $18,315(6.20)$ & $36,033(6.27)$ & $50,899(7.18)$ & $66,460(6.47)$ & $62,901(6.03)$ \\
\hline Immunosuppressants & II,700 (3.96) & $20,876(3.63)$ & $27,833(3.92)$ & $81,944(7.98)$ & $49,680(4.76)$ \\
\hline $\begin{array}{l}\text { Drugs for peptic ulcer and gastro-oesophageal } \\
\text { reflux disease }\end{array}$ & II,004 (3.72) & $23,705(4.13)$ & $29,663(4.18)$ & $35,153(3.42)$ & $69,766(6.69)$ \\
\hline Antidepressants & $13,138(4.45)$ & $25,212(4.39)$ & $30,028(4.23)$ & $40,596(3.95)$ & $42,412(4.07)$ \\
\hline Opioids & I3,543 (4.58) & $22,773(3.96)$ & $24,895(3.51)$ & $35,922(3.50)$ & $40,086(3.84)$ \\
\hline Antiepileptics & II,343 (3.84) & $20,513(3.57)$ & $24,91 \mathrm{I}(3.5 \mathrm{I})$ & $36,384(3.55)$ & $35,708(3.42)$ \\
\hline Lipid modifying agents, plain & $8912(3.02)$ & $19,429(3.38)$ & $25,389(3.58)$ & $30,908(3.01)$ & $35,723(3.43)$ \\
\hline Antipsychotics & $7548(2.55)$ & I3,737 (2.39) & $15,886(2.24)$ & $33,627(3.28)$ & $27,317(2.62)$ \\
\hline First-generation & $2007(0.68)$ & $3731(0.65)$ & $4049(0.57)$ & $6791(0.66)$ & $7112(0.68)$ \\
\hline Second-generation & $554 \mathrm{I}(\mathrm{I} .87)$ & $10,006(1.74)$ & II,837 (1.67) & $26,836(2.61)$ & 20,205 (1.94) \\
\hline Antineoplastic agents & $9372(3.17)$ & $|6,5| \mid(2.87)$ & $18,634(2.63)$ & $46,176(4.50)$ & $30,173(2.90)$ \\
\hline Chemotherapy & $4712(1.59)$ & 7415 (1.29) & $6830(0.96)$ & $16,776(1.63)$ & $12,036(1.16)$ \\
\hline Targeted therapy & $4660(1.58)$ & $9096(1.58)$ & II,804 (1.66) & $29,400(2.86)$ & $18,137(1.74)$ \\
\hline Anxiolytics & $7968(2.70)$ & $13,246(4.48)$ & $14,402(2.03)$ & $19,534(1.90)$ & $22,351(2.14)$ \\
\hline Direct acting antivirals & $4368(1.48)$ & 11,157 (1.94) & $11,323(1.60)$ & $15,054(1.47)$ & $13,263(1.27)$ \\
\hline
\end{tabular}

Note: Values are reported as $\mathrm{n}(\%)$.

Abbreviation: FAERS, Food and Drug Administration Adverse Event Reporting System. 


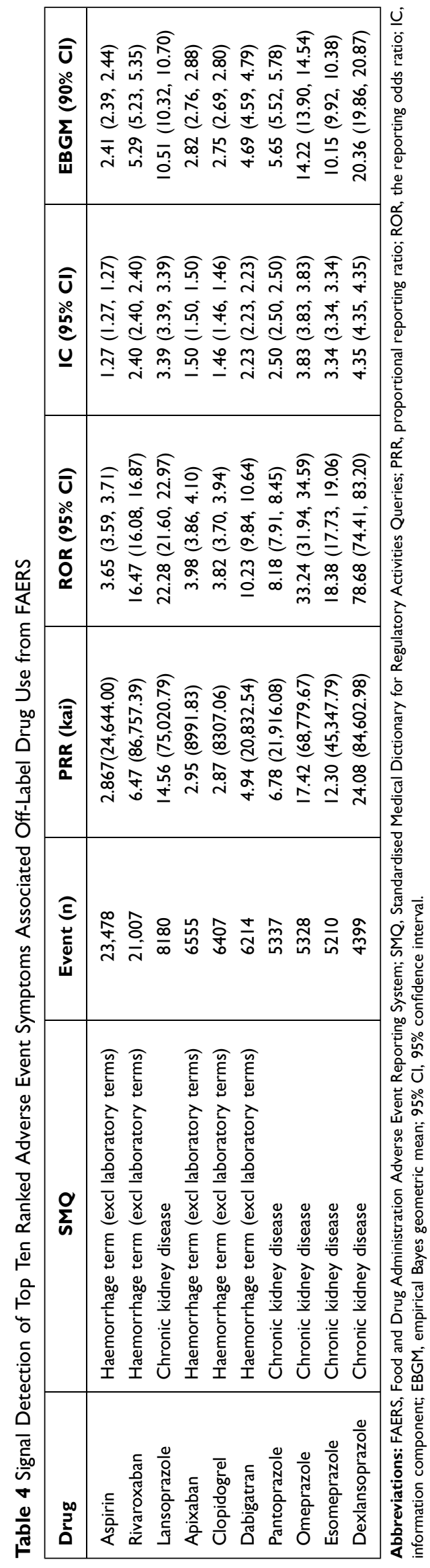

$21.57 \%$ ( 88 out of 1134 ), while those 65 and older ranged from $12.08 \%$ (137 out of 1134 ) to $27.02 \%$ (207 out of 766 ). In addition, 2.80 to 3.14 for AEs on average were reported per patient who used off-label drugs, and these patients were coadministered 5.02 to 5.71 other drugs on average. The most frequent outcome was "requires hospitalization or extension of hospital stay" (28.85-48.31\%), followed by "death" (7.25$17.87 \%)$.

\section{AEs Related to Off-Label Drugs in KIDS-KD}

In the KIDS-KD data, secondary terms-events $(43.45 \%$ to $48.62 \%)$ such as "inappropriate schedule of drug administration", "medication error", and "surgical intervention" were the most common AEs from 2014 to 2018 (Table 6). The next common SOC was body as a whole-general disorders $(8.97 \%$ to $11.80 \%)$ such as effect, lack of, drug ineffective, and pain from 2014 to 2018, but not 2016.

\section{Off-Label Drugs Related to Reported AEs in KIDS-KD}

In the KIDS-KD data, AEs related to administered single offlabel drugs were used for a further analysis. Immunosuppressants were the most common AE-related drugs reported for 2014 (63 out of 307, 20.52\%) and 2015 ( 66 out of $436,15.14 \%$ ), while antipsychotics were the most common AE-related drugs reported for 2016 (421 out of $1007,41.81 \%$ ) (Table 7). Parathyroid hormones and analogues were the most common drugs related to $\mathrm{AE}$ reported in 2017 (122 out of 513, 23.78\%), while other antineoplastic agents were the most common for 2018 (148 out of 733, $20.19 \%$ ) (Table 7). The frequencies of other antineoplastic agents $(0.65 \%$ to $20.19 \%)$ and direct acting antivirals $(0 \%$ to 16.92\%) trended to increase from 2014 to 2018.

\section{Discussion}

Scientific evidence related to off-label prescribing is scant compared to that related to prescriptions for approved indications, indicating that safety issues related to these drugs are a concern. Spontaneous reports can be used to determine AEs associated with off-label drug use. In relation to this, this study analyzed AEs associated with offlabel drug use using data from FAERS and KIDS-KD.

FAERS is frequently exploited to perform disproportionality analyses given its quick updates and the fact that it contains data reported by multiple countries even though it is operated by the US FDA. ${ }^{36,37}$ KIDS-KD is managed by the 
Table 5 Demographic Characteristics of the Subjects from the KIDS-KD Data

\begin{tabular}{|c|c|c|c|c|c|}
\hline Characteristics & 2014 & 2015 & 2016 & 2017 & 2018 \\
\hline Number of patients, $n$ & 408 & 544 & 1193 & 766 & 1134 \\
\hline Age, years & $52.72 \pm 22.25$ & $46.62 \pm 24.71$ & $59.86 \pm 21.45$ & $57.06 \pm 21.50$ & $50.10 \pm 20.70$ \\
\hline Less than 28 days old, $\mathrm{n}$ (\%) & $\mathrm{I}(0.25)$ & 0 & $3(0.25)$ & $2(0.26)$ & $9(0.79)$ \\
\hline 28 days to 2 years, $\mathrm{n}(\%)$ & $6(1.47)$ & $29(5.33)$ & $9(0.75)$ & II (I.44) & $26(2.29)$ \\
\hline 2 to 12 years, $\mathrm{n}(\%)$ & $16(3.92)$ & $23(4.23)$ & $20(1.68)$ & $20(2.6 I)$ & $30(2.65)$ \\
\hline 12 to 19 years, $\mathrm{n}(\%)$ & $10(2.45)$ & $24(4.4 I)$ & $16(1.34)$ & $17(2.22)$ & $23(2.03)$ \\
\hline 19 to 64 years old, $\mathrm{n}(\%)$ & $133(32.60)$ & $172(31.62)$ & $216(18.11)$ & $242(31.59)$ & $315(27.78)$ \\
\hline 65 years old or older, $\mathrm{n}(\%)$ & $77(18.87)$ & $85(15.63)$ & $248(20.79)$ & $207(27.02)$ & $137(12.08)$ \\
\hline Unknown, n (\%) & $165(40.44)$ & $211(38.79)$ & $68 \mathrm{I}(57.08)$ & $267(34.86)$ & $594(52.38)$ \\
\hline \multicolumn{6}{|l|}{ Gender (n, \%) } \\
\hline Male, n (\%) & $184(45.10)$ & $203(37.32)$ & $282(23.64)$ & $277(36.16)$ & $494(43.56)$ \\
\hline Female, n (\%) & $190(46.57)$ & $260(47.98)$ & $405(33.95)$ & $383(50.00)$ & $456(40.21)$ \\
\hline Unknown, n (\%) & $34(8.33)$ & $80(\mid 4.7 I)$ & $506(42.4 I)$ & $106(13.84)$ & $184(16.23)$ \\
\hline Coadministration drugs, $\mathrm{n}$ & $5.03 \pm 4.19$ & $5.41 \pm 4.70$ & $5.65 \pm 4.79$ & $5.02 \pm 4.40$ & $5.7 I \pm 4.8 I$ \\
\hline Reported events per patients, $\mathrm{n}$ & $2.85 \pm 3.47$ & $2.80 \pm 3.52$ & $2.90 \pm 3.70$ & $2.95 \pm 3.51$ & $3.14 \pm 4.20$ \\
\hline Number of outcomes, $n$ & 89 & 88 & 207 & 334 & 610 \\
\hline Results in persistent or significant disability or incapacity, $\mathrm{n}(\%)$ & $\mathrm{I}(1.12)$ & 0 & $2(0.08)$ & 0 & $4(0.66)$ \\
\hline Congenital anomaly/birth defect, n (\%) & 0 & 0 & 0 & 0 & $7(1.15)$ \\
\hline Life-threatening, n (\%) & $\mathrm{I}(\mathrm{I} .12)$ & I (I.I4) & $4(1.93)$ & $9(2.69)$ & $8(1.31)$ \\
\hline Death, n (\%) & $10(11.24)$ & $12(13.64)$ & $15(7.25)$ & $29(8.68)$ & $109(17.87)$ \\
\hline Requires hospitalization or extension of hospital stay, $\mathrm{n}(\%)$ & $43(48.31)$ & $28(31.82)$ & $74(35.75)$ & $115(34.43)$ & $176(28.85)$ \\
\hline Others, n (\%) & $34(38.20)$ & $47(53.4 I)$ & $112(54.11)$ & $181(54.19)$ & $306(50.16)$ \\
\hline
\end{tabular}

Note: Values are reported as $\mathrm{n}(\%)$ or mean \pm standard deviation.

Abbreviation: KIDS-KD, the Korea Institute of Drug Safety \& Risk Management (Ministry of Food and Drug Safety)-Korea Adverse Event Reporting System database.

KIDS, and studies using that system have increased. ${ }^{38,39}$ The reports on specific AEs in FAERS use MedDRA, while those in KIDS-KD use WHOART. We exploited these two databases as to whether these two terminologies can be used for the detection of off-label use.

Although the occurrence rate of AEs and adverse symptoms varied and depended on the off-label drugs used, the occurrence rates of reported AEs increased according to both FAERS and KIDS-KD from 2014 to 2018. Higher rates of AEs were reported for women than for men in our study, consistent with previous reports indicating higher rates for women than men. ${ }^{40,41}$

The frequency of off-label administration can vary depending on the age and gender of patients and the drugs they use. The frequency of off-label medicines was highest in less than 48.5 years old patients and decreased in older patients according to the Medical Office of XXI Century electronic health record network in Canada. ${ }^{6}$ The frequency of receiving an off-label prescription was reduced for each year of increase in age up to 20 years. ${ }^{42}$ In contrast, an increase in off-label antidepressant use was found in patients aged 0 to 17 years. ${ }^{43}$ It is well known that the use of off-label drugs in children is both common and associated with the risk of adverse drug reactions. ${ }^{44}$ However, our results showed that the $\mathrm{AE}$ frequencies in adults were higher compared to those in children. The severity and types of AEs can vary depending on the physiological conditions of the patients such as their sex, age, and body weight. ${ }^{45}$ Speculation is permitted given that both monitoring systems underreported due to a failure to recognize an $\mathrm{AE}$ or a failure to report a recognized $\mathrm{AE}$ in children. ${ }^{46,47}$

Additionally, reported AEs associated with off-label drug use were common among older patients, most likely related to their complex patterns of medication administration and high rates of medical comorbidity. ${ }^{9,17}$ Specifically, the frequencies of reported AEs associated with off-label drug use among older patients tended to increase from 2014 to 2018 in KIDS-KD, possibly due to the increase in the aged population. Older adults are especially vulnerable, as age-related physiologic changes, coexisting conditions, and instances of polypharmacy all increase the risk of an $\mathrm{AE}$ and the health 
Table 6 Top Ten Adverse Event Symptoms of Off-Label Drug Use from the KIDS-KD Data

\begin{tabular}{|c|c|c|c|c|c|}
\hline SOC & $\begin{array}{c}2014 \\
(N=1068)\end{array}$ & $\begin{array}{c}2015 \\
(N=1355)\end{array}$ & $\begin{array}{c}2016 \\
(N=2818)\end{array}$ & $\begin{array}{c}2017 \\
(N=2040)\end{array}$ & $\begin{array}{c}2018 \\
(N=2885)\end{array}$ \\
\hline Secondary terms-events & $464(43.45)$ & $609(44.94)$ & $1370(48.62)$ & $910(44.61)$ & $1388(48.11)$ \\
\hline Body as a whole-general disorders & $126(11.80)$ & $159(11.73)$ & $238(8.45)$ & $183(8.97)$ & $289(10.02)$ \\
\hline Gastro-intestinal system disorders & $64(5.99)$ & $90(6.64)$ & $254(9.01)$ & $174(8.53)$ & $182(6.31)$ \\
\hline Central \& peripheral nervous system disorders & $76(7.12)$ & $80(5.90)$ & $222(7.88)$ & $124(6.08)$ & $110(3.81)$ \\
\hline Psychiatric disorders & $47(4.40)$ & $57(4.21)$ & II4 (4.05) & $89(4.36)$ & $114(3.95)$ \\
\hline Application site disorders & $20(1.87)$ & $68(5.02)$ & $|3|(4.65)$ & $69(3.38)$ & $100(3.47)$ \\
\hline Skin and appendages disorders & $62(5.8 \mathrm{I})$ & $57(4.21)$ & $87(3.09)$ & $58(2.84)$ & $87(3.02)$ \\
\hline Respiratory system disorders & $31(2.90)$ & $27(1.99)$ & $34(1.21)$ & $61(2.99)$ & $84(2.91)$ \\
\hline Musculo-skeletal system disorders & $15(1.40)$ & $28(2.07)$ & $60(2.13)$ & $43(2.11)$ & $58(2.01)$ \\
\hline Metabolic and nutritional disorders & $9(0.84)$ & $23(1.70)$ & $41(1.45)$ & $48(2.35)$ & $49(1.70)$ \\
\hline
\end{tabular}

Notes: Values are reported as $\mathrm{n}(\%)$. Secondary terms-events mainly contained inappropriate schedule of drug administration, medication error, incorrect technique in drug usage process, incorrect dose prescribed, incorrect dose administered, and surgical intervention.

Abbreviations: KIDS-KD, the Korea Institute of Drug Safety \& Risk Management (Ministry of Food and Drug Safety)-Korea Adverse Event Reporting System database; SOC, System Organ Classes.

Table 7 Off-Label Single-Drug Classifications for Adverse Events in the KIDS-KD Data

\begin{tabular}{|c|c|c|c|c|c|}
\hline Drug Classification & $2014(N=307)$ & $2015(N=436)$ & $2016(N=1007)$ & $2017(N=5 \mid 3)$ & $2018(N=733)$ \\
\hline Second generation antipsychotics & I (0.33) & $7(1.61)$ & $42 I(4 I .8 I)$ & 21 (4.09) & $13(1.77)$ \\
\hline Parathyroid hormones and analogues & $8(2.61)$ & $58(13.30)$ & $242(24.03)$ & $122(23.78)$ & $14(1.91)$ \\
\hline Antineoplastic agents & $2(0.65)$ & $29(6.65)$ & 37 (3.67) & $49(9.55)$ & $148(20.19)$ \\
\hline Chemotherapy & 0 & $6(1.38)$ & $8(0.79)$ & II (2.14) & II (I.50) \\
\hline Targeted therapy & $2(0.65)$ & $26(5.96)$ & $30(2.98)$ & $49(9.55)$ & $148(20.19)$ \\
\hline Immunosuppressants & $63(20.52)$ & $66(15.14)$ & $26(2.58)$ & $43(8.38)$ & $49(6.68)$ \\
\hline Direct acting antivirals & 0 & $2(0.46)$ & $13(1.29)$ & $23(4.48)$ & $124(16.92)$ \\
\hline Hormonal contraceptives for systemic use & $43(14.01)$ & $16(3.67)$ & $14(1.39)$ & $19(3.70)$ & $9(1.23)$ \\
\hline Antiepileptics & $7(2.28)$ & $16(3.67)$ & $37(3.67)$ & $16(3.12)$ & $20(2.73)$ \\
\hline Drugs used in benign prostatic hypertrophy & $24(7.82)$ & $16(3.67)$ & $13(1.29)$ & $9(1.75)$ & $13(1.77)$ \\
\hline Antiinfectives and antiseptics, excl. Combinations & $47(|4.3|)$ & $10(2.29)$ & 0 & $4(0.78)$ & $6(0.82)$ \\
\hline Antithrombotic agents & $2(0.65)$ & II (2.52) & $21(2.09)$ & $23(4.48)$ & $4(0.55)$ \\
\hline Antidepressants & $5(1.63)$ & $7(1.6 I)$ & $7(0.70)$ & $3(0.58)$ & $14(1.91)$ \\
\hline
\end{tabular}

Note: Values are reported as $\mathrm{n}(\%)$.

Abbreviation: KIDS-KD, the Korea Institute of Drug Safety \& Risk Management (Ministry of Food and Drug Safety)-Korea Adverse Event Reporting System database.

impact of experiencing one. A survey taken in the United States showed that nearly $20 \%$ of participants aged 65 years and older took at least five drugs per week. ${ }^{48,49}$ Polypharmacy increases the risk of drug-drug interactions and AEs, which can cause hospitalization. ${ }^{48,49}$

Gastrointestinal nonspecific symptoms and therapeutic procedures were the most commonly reported AEs in FAERS. Gastrointestinal symptoms induced by medication can frequently occur. ${ }^{50}$ Medications can produce symptoms due to changes in gastrointestinal physiology (eg, anticholinergics) or intestinal microbiota (eg, antibiotics), damage to tissues (eg, non-steroidal anti-inflammatory drugs), or through unknown mechanisms. ${ }^{51,52}$ The frequencies of secondary-term events including "medication error", "inappropriate schedule of drug administration", and "drug ineffective" were higher in the KIDS-KD data. This suggests that the effectiveness of off-label drugs and issues related to medication errors must be considered. The differences in the results from FAERS and KIDS$\mathrm{KD}$ may have been caused by differences in the $\mathrm{AE}$ reporting systems, including the $\mathrm{AE}$ terminology or regulations pertaining to off-label use.

Antithrombotic agents were the most frequently reported AE-associated therapeutic class of drugs in FAERS, similar to a previous report and signs of hemorrhaging were most frequently reported for antithrombotic agents. ${ }^{53}$ Anticoagulants, opioids, and diabetic agents were high-priority drug class targets for AE prevention efforts 
by the Department of Health and Human Services in $2019 .{ }^{53}$ Recently, the adverse signal of chronic kidney disease associated with proton pump inhibitors based on FAERS has been reported. ${ }^{54}$ This result was similar to the results here. The off-label prescribing of proton pump inhibitors has been widely reported, including in patients taking antiplatelet, anticoagulant, or corticosteroid drugs, for the prevention of chemotherapy or radiotherapyassociated lesions in patients with cancer, or in the management of extra-digestive symptoms. ${ }^{55}$ The number of reported AEs related to new drug categories, such as other antineoplastic agents, tended to increase from 2014 to 2018 in both the FAERS and KIDS-KD data because possibly approvals of these types of medicines increased dramatically. ${ }^{56}$

In our study, we used four different data mining algorithm for signal detection using the FAERS data because the detectability depends on the effect sizes and the level of confounding data present in the data. ${ }^{57}$ There is no gold standard for this type of algorithm, and various disproportionality analysis methods arrive at different concordance under different circumstances. ${ }^{58}$ On the other hand, the disproportional analysis could not be conducted using KIDS-KD data in our study because the whole reports were not provided according to the policy of KIDS.

Another limitation of this study was that the AE reports in the spontaneous AE systems were submitted voluntarily, meaning that underreporting may be an issue. Specifically, there were relatively few reported $\mathrm{AE}$ cases associated with off-label drugs in KIDS-KD. Although the duplicated reports were removed according to the same report date and CASEID, some duplicated reports may remain. The reported AEs are unable to determine causal associations and cannot be verified, meaning that this information cannot be used to infer incidence rates. As a result, offlabel status in this study was not verified.

However, pharmacoepidemiologic research on adverse drug effects, especially in relation to off-label drug use, is difficult due to limited sample sizes, reduced follow-up rates, and evaluations of surrogate markers. ${ }^{59}$ There are no tools or methodologies that are specific to the monitoring of the safety of medicines that are used as off-label drugs. To the best of the author's knowledge, this study addressed the adverse events of off-label use for the first time through AE reporting system databases in a comprehensive manner. Relatively few studies have recently described AEs associated with off-label uses in limited populations ${ }^{60}$ or specific cases. ${ }^{61}$ The results here can be useful when making predictions of AEs associated with off-label use. However, off-label use does not necessarily mean that a drug is being used inappropriately. It is believed that the tools and methodologies for the postmarket safety monitoring of approved drugs can generally be used to monitor the safety of medicines used in an offlabel manner. Another limitation of this study was that it was difficult to distinguish between off-label drugs and other co-administered drugs because off-drug use is included within the adverse-drug reaction symptom terms in the KIDS-KD system. Therefore, only single administration of off-label drugs was analyzed in our study.

It is known that off-label drug use is a risk factor for AEs. ${ }^{17}$ In this study, we investigated adverse symptoms and trends related to the use of off-label drugs using data from spontaneous AE reporting systems. We believe that the collection and analysis of these real-world data may pay attention to healthcare professionals as a point of view of the clinical implications of off-label drug use. Further study is still needed to evaluate the clinical benefits and risks of off-label drug uses.

\section{Conclusion}

AEs associated with off-label drugs were analyzed using data from adverse event reporting systems in this study. The numbers of reported AEs with off-label drug increased annually. AEs associated with off-label drugs may have a significant impact on older patients. From the findings here, older patients should be monitored carefully for AEs when treated with off-label drugs. Healthcare experts should potentially be concerned about the prescription of off-label drugs, especially anticoagulants and newly developed drugs such as immunosuppressants and antineoplastic agents.

\section{Acknowledgments}

This study was supported by a grant (19172MFDS130) from the Ministry of Food and Drug Safety and the National Research Foundation of Korea grant funded by the Korea government (MSIT) (2021R1A2C1006046). We would like to acknowledge FDA Adverse Event Reporting System and KIDS-KAERS database (KIDS-KD), Korea Institute of Drug Safety \& Risk Management (Ministry of Food and Drug Safety) for providing the data.

\section{Disclosure}

The authors report no conflicts of interest in this work. 


\section{References}

1. Aronson JK, Ferner RE. Unlicensed and off-label uses of medicines: definitions and clarification of terminology. Br J Clin Pharmacol. 2017;83(12):2615-2625. doi:10.1111/bcp.13394

2. Balan S, Hassali MAA, Mak VSL. Two decades of off-label prescribing in children: a literature review. World J Pediatr. 2018;14 (6):528-540. doi:10.1007/s12519-018-0186-y

3. Corny J, Lebel D, Bailey B, Bussieres JF. Unlicensed and off-label drug use in children before and after pediatric governmental initiatives. J Pediatr Pharmacol Ther. 2015;20(4):316-328.

4. Hoon D, Taylor MT, Kapadia P, Gerhard T, Strom BL, Horton DB. Trends in off-label drug use in ambulatory settings: 2006-2015. Pediatrics. 2019;144(4):e20190896. doi:10.1542/ peds.2019-0896

5. Alexander GC, Gallagher SA, Mascola A, Moloney RM, Stafford RS. Increasing off-label use of antipsychotic medications in the United States, 1995-2008. Pharmacoepidemiol Drug Saf. 2011;20(2):177-184. doi:10.1002/pds.2082

6. Eguale T, Buckeridge DL, Winslade NE, Benedetti A, Hanley JA, Tamblyn R. Drug, patient, and physician characteristics associated with off-label prescribing in primary care. Arch Intern Med. 2012;172 (10):781-788. doi:10.1001/archinternmed.2012.340

7. Radley DC, Finkelstein SN, Stafford RS. Off-label prescribing among office-based physicians. Arch Intern Med. 2006;166 (9):1021-1026. doi:10.1001/archinte.166.9.1021

8. Golchin N, Frank SH, Vince A, Isham L, Meropol SB. Polypharmacy in the elderly. J Res Pharm Pract. 2015;4(2):85-88. doi:10.4103/ 2279-042X.155755

9. Jackson SH, Jansen PA, Mangoni AA. Off-label prescribing in older patients. Drugs Aging. 2012;29(6):427-434. doi:10.2165/11633520000000000-00000

10. Kumar Kakkar A, Dahiya N. The evolving drug development landscape: from blockbusters to niche busters in the orphan drug space. Drug Dev Res. 2014;75(4):231-234. doi:10.1002/ddr.21176

11. Scannell JW, Blanckley A, Boldon H, Warrington B. Diagnosing the decline in pharmaceutical R\&D efficiency. Nat Rev Drug Discov. 2012;11(3):191-200. doi:10.1038/nrd3681

12. Gibson S, Raziee HR, Lemmens T. Why the shift? Taking a closer look at the growing interest in niche markets and personalized medicine. World Med Health Policy. 2015;7(1):3-27. doi:10.1002/wmh3.131

13. Mingorance A. Drivers of orphan drug development. ACS Med Chem Lett. 2018;9(10):962-964. doi:10.1021/acsmedchemlett.8b00438

14. Zhao S, Nair JR, Moots RJ. Biosimilars: from extrapolation into off label use. Curr Pharm Des. 2017;23(44):6746-6751. doi:10.2174/ 1381612824666171129193258

15. Bloom BE. Recent successes and future predictions on drug repurposing for rare diseases. Expert Opin Orphan Drugs. 2016;4(1):1-4. doi: $10.1517 / 21678707.2016 .1120664$

16. Cohen JP, Awatin JG. Patient access to orphan drugs. Expert Opin Orphan Drugs. 2017;5(12):923-932. doi:10.1080/21678707.2017.1402676

17. Eguale T, Buckeridge DL, Verma A, et al. Association of off-label drug use and adverse drug events in an adult population. JAMA Intern Med. 2016;176(1):55-63. doi:10.1001/jamainternmed.2015.6058

18. Shapiro S. Appetite-suppressant drugs and valvular heart disease. $N$ Engl J Med. 1999;340(6):477-478; author reply 478-480.

19. Fournier A, Zureik M. Estimate of deaths due to valvular insufficiency attributable to the use of benfluorex in France. Pharmacoepidemiol Drug Saf. 2012;21(4):343-351. doi:10.1002/pds.3213

20. Sakaeda T, Tamon A, Kadoyama K, Okuno Y. Data mining of the public version of the FDA adverse event reporting system. Int $J$ Med Sci. 2013;10(7):796-803. doi:10.7150/ijms.6048

21. Stricker BH, Psaty BM. Detection, verification, and quantification of adverse drug reactions. BMJ. 2004;329(7456):44-47. doi:10.1136/ bmj.329.7456.44
22. U.S.Food \& Drug Administration. FDA adverse event reporting system (FAERS): latest quarterly data files. Available from: https:// www.fda.gov/Drugs/GuidanceComplianceRegulatoryInformation/ Surveillance/AdverseDrugEffects/ucm082193.htm. Accessed August 12, 2021.

23. Korea Institute of Drug Safety and Risk Management. User guide: Korea institute of drug safety and risk management-Korea adverse event reporting system database (KIDS-KD). Available from: https:// www.drugsafe.or.kr/iwt/ds/ko/openinfo/DrugAdrDataProc.do. Accessed August 12, 2021.

24. ICH Legal Mention. MedDRA. Medical dictionary for regulatory activities. Available from: https://www.meddra.org/. Accessed August 12, 2021.

25. Uppsala Monitoring Center. The WHO-ART adverse reaction terminology. Available from: http://www.umc-products.com/gra phics/3149.pdf. Accessed August 12, 2021.

26. Beyzarov E, Chen Y, Julg R, et al. Global safety database summary of COVID-19-related drug utilization-safety surveillance: a sponsor's perspective. Drug Saf. 2021;44(1):95-105. doi:10.1007/s40264-02001035-x

27. U. S. Food \& Drug Administration. CFR-code of federal regulations title 21. sec 201.57 (c) (9) (iv) (4). Available from: https://www. accessdata.fda.gov/scripts/cdrh/cfdocs/cfcfr/cfrsearch.cfm?fr=201.57. Accessed August 12, 2021.

28. ICH Harmonised Guideline. Addendum To ICH E11: clinical investigation of medicinal products in the pediatric population E11 (R1). Available from: https://www.meddra.org/. Accessed August 12, 2021.

29. Mozzicato P. Standardised MedDRA queries: their role in signal detection. Drug Saf. 2007;30(7):617-619. doi:10.2165/00002018200730070-00009

30. Fan Q, Hu Y, Yang C, Zhao B. Myocarditis following the use of different immune checkpoint inhibitor regimens: a real-world analysis of post-marketing surveillance data. Int Immunopharmacol. 2019;76:105866. doi:10.1016/j.intimp.2019.105866

31. Evans SJ, Waller PC, Davis S. Use of proportional reporting ratios (PRRs) for signal generation from spontaneous adverse drug reaction reports. Pharmacoepidemiol Drug Saf. 2001;10(6):483-486. doi:10.1002/pds. 677

32. van Puijenbroek EP, Bate A, Leufkens HG, Lindquist M, Orre R, Egberts AC. A comparison of measures of disproportionality for signal detection in spontaneous reporting systems for adverse drug reactions. Pharmacoepidemiol Drug Saf. 2002;11(1):3-10. doi:10.1002/pds. 668

33. Bate A, Lindquist M, Edwards IR, et al. A Bayesian neural network method for adverse drug reaction signal generation. Eur $J$ Clin Pharmacol. 1998;54(4):315-321. doi:10.1007/s002280050466

34. Duggirala HJ, Tonning JM, Smith E, et al. Use of data mining at the food and drug administration. $J$ Am Med Inform Assoc. 2016;23 (2):428-434. doi:10.1093/jamia/ocv063

35. Szarfman A, Machado SG, O'Neill RT. Use of screening algorithms and computer systems to efficiently signal higher-than-expected combinations of drugs and events in the US FDA's spontaneous reports database. Drug Saf. 2002;25(6):381-392. doi:10.2165/00002018200225060-00001

36. Cepaityte D, Siafis S, Papazisis G. Safety of antipsychotic drugs: a systematic review of disproportionality analysis studies. Behav Brain Res. 2021;404:113168. doi:10.1016/j.bbr.2021.113168

37. Raschi E, Gatti M, Gelsomino F, Ardizzoni A, Poluzzi E, De Ponti F. Lessons to be learnt from real-world studies on immune-related adverse events with checkpoint inhibitors: a clinical perspective from pharmacovigilance. Target Oncol. 2020;15(4):449-466. doi:10.1007/s11523-020-00738-6

38. Choi JY, Choi JH, Kim MG, Rhie SJ. Signal detection of adverse drug reactions of cephalosporins using data from a national pharmacovigilance database. Pharmaceuticals (Basel). 2021;14(5):425. doi: $10.3390 / \mathrm{ph} 14050425$ 
39. Oh IS, Baek YH, Kim HJ, Lee M, Shin JY. Differential completeness of spontaneous adverse event reports among hospitals/clinics, pharmacies, consumers, and pharmaceutical companies in South Korea. PLoS One. 2019;14(2):e0212336. doi:10.1371/journal.pone.0212336

40. Martin RM, Biswas PN, Freemantle SN, Pearce GL, Mann RD. Age and sex distribution of suspected adverse drug reactions to newly marketed drugs in general practice in England: analysis of 48 cohort studies. Br J Clin Pharmacol. 1998;46(5):505-511. doi:10.1046/ j.1365-2125.1998.00817.x

41. Pouyanne P, Haramburu F, Imbs JL, Begaud B. Admissions to hospital caused by adverse drug reactions: cross sectional incidence study. French pharmacovigilance centres. BMJ. 2000;320 (7241):1036. doi:10.1136/bmj.320.7241.1036

42. Allen HC, Garbe MC, Lees J, et al. Off-label medication use in children, more common than we think: a systematic review of the literature. J Okla State Med Assoc. 2018;111(8):776-783.

43. Schroder C, Dorks M, Kollhorst B, et al. Extent and risks of antidepressant off-label use in children and adolescents in Germany between 2004 and 2011. Pharmacoepidemiol Drug Saf. 2017;26 (11):1395-1402. doi:10.1002/pds.4289

44. Bellis JR, Kirkham JJ, Thiesen S, et al. Adverse drug reactions and off-label and unlicensed medicines in children: a nested case-control study of inpatients in a pediatric hospital. BMC Med. 2013;11(1):238. doi:10.1186/1741-7015-11-238

45. Mizutani S, Noro Y, Kotera M, Goto S. Pharmacoepidemiological characterization of drug-induced adverse reaction clusters towards understanding of their mechanisms. Comput Biol Chem. 2014;50:50-59. doi:10.1016/j.compbiolchem.2014.01.006

46. Hawcutt DB, Russell NJ, Maqsood H, et al. Spontaneous adverse drug reaction reports for neonates and infants in the UK 2001-2010: content and utility analysis. Br J Clin Pharmacol. 2016;82 (6):1601-1612. doi:10.1111/bcp.13067

47. Lopez-Gonzalez E, Herdeiro MT, Figueiras A. Determinants of under-reporting of adverse drug reactions: a systematic review. Drug Saf. 2009;32(1):19-31. doi:10.2165/00002018-20093201000002

48. Kaufman DW, Kelly JP, Rosenberg L, Anderson TE, Mitchell AA. Recent patterns of medication use in the ambulatory adult population of the United States: the Slone survey. JAMA. 2002;287(3):337-344. doi:10.1001/jama.287.3.337

49. Salvi F, Marchetti A, D’Angelo F, Boemi M, Lattanzio F, Cherubini A. Adverse drug events as a cause of hospitalization in older adults. Drug Saf. 2012;35(Suppl 1):29-45. doi:10.1007/ BF03319101
50. Philpott HL, Nandurkar S, Lubel J, Gibson PR. Drug-induced gastrointestinal disorders. Frontline Gastroenterol. 2014;5(1):49-57. doi:10.1136/flgastro-2013-100316

51. Dubeau MF, Iacucci M, Beck PL, et al. Drug-induced inflammatory bowel disease and IBD-like conditions. Inflamm Bowel Dis. 2013;19 (2):445-456. doi:10.1002/ibd.22990

52. Fosnes GS, Lydersen S, Farup PG. Constipation and diarrhoea common adverse drug reactions? A Cross Sectional Study in the general population. BMC Clin Pharmacol. 2011;11(1):2. doi:10.1186/1472-6904-11-2

53. U.S. Department of Health and Human Services. National action plan for adverse drug event prevention. Available from: https://health.gov/ hcq/pdfs/ade-action-plan-508c.pdf. Accessed August 12, 2021.

54. Wu B, Li D, Xu T, Luo M, He Z, Li Y. Proton pump inhibitors associated acute kidney injury and chronic kidney disease: data mining of US FDA adverse event reporting system. Sci Rep. 2021;11(1):3690. doi:10.1038/s41598-021-83099-y

55. Lassalle M, Le Tri T, Bardou M, et al. Use of proton pump inhibitors in adults in France: a Nationwide Drug Utilization Study. Eur J Clin Pharmacol. 2020;76(3):449-457. doi:10.1007/s00228-019-02810-1

56. Kaplon H, Reichert JM. Antibodies to watch in 2019. MAbs. 2019;11 (2):219-238. doi:10.1080/19420862.2018.1556465

57. Dijkstra L, Garling M, Foraita R, Pigeot I. Adverse drug reaction or innocent bystander? A systematic comparison of statistical discovery methods for spontaneous reporting systems. Pharmacoepidemiol Drug Saf. 2020;29(4):396-403. doi:10.1002/pds.4970

58. Hou Y, Ye X, Wu G, Cheng G, Du X, He J. A comparison of disproportionality analysis methods in national adverse drug reaction databases of China. Expert Opin Drug Saf. 2014;13(7):853-857. doi:10.1517/14740338.2014.915938

59. Dal Pan GJ. Monitoring the safety of medicines used off-label. Clin Pharmacol Ther. 2012;91(5):787-795. doi:10.1038/clpt.2012.24

60. Mascolo A, Scavone C, Bertini M, et al. Safety of anticancer agents used in children: a focus on their off-label use through data from the spontaneous reporting system. Front Pharmacol. 2020;11:621. doi:10.3389/fphar.2020.00621

61. Li H, Deng J, Yu P, Deng L, Ren X. Gaining insight into irrational off-label use of vidarabine through analysis of a spontaneous reporting system in China. J Clin Pharm Ther. 2020;45(6):1301-1311. doi:10.1111/jcpt.13209
Therapeutics and Clinical Risk Management

\section{Publish your work in this journal}

Therapeutics and Clinical Risk Management is an international, peerreviewed journal of clinical therapeutics and risk management, focusing on concise rapid reporting of clinical studies in all therapeutic areas, outcomes, safety, and programs for the effective, safe, and sustained use of medicines. This journal is indexed on PubMed Central, CAS,

\section{Dovepress}

EMBase, Scopus and the Elsevier Bibliographic databases. The manuscript management system is completely online and includes a very quick and fair peer-review system, which is all easy to use. Visit http://www.dovepress.com/testimonials.php to read real quotes from published authors. 